\title{
Propofol-Infusionssyndrom bei Behandlung eines Status epilepticus
}

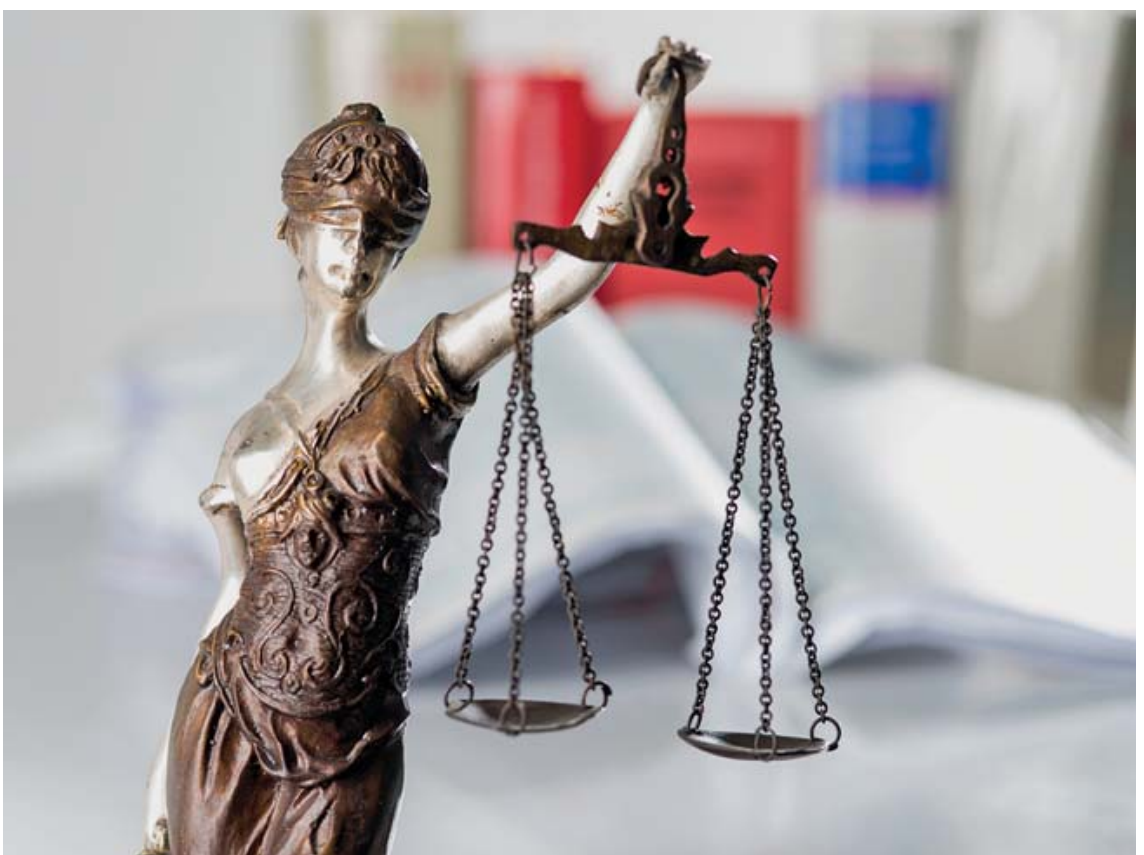

Schlichtungsstellen für Arzthaftpflichtfragen bieten Patienten, Ärzten und Versicherern eine Möglichkeit, Arzthaftungsstreitigkeiten außergerichtlich zu klären. In der Rubrik „Fälle der Schlichtungsstelle“ stellen wir abgeschlossene Fälle aus der Schlichtungsstelle für Arzthaftpflichtfragen der norddeutschen Ärztekammern vor. Quelle: KH Krauskopf.

\section{Der Fall}

Der 17-jährige Patient bekommt beim Sport einen Medizinball an den Kopf. Nach kurzer Benommenheit kann er das Training fortsetzen, später werden jedoch eine Hyperventilation und ein 10 min lang dauernder Krampfanfall beobachtet. Nach der Versorgung vom Notarzt mit $10 \mathrm{mg}$ Midazolam wird er in die Klinik transportiert, wo es in der Zentralen Notaufnahme erneut zu generalisierten Krampfanfallen kommt, die mit Midazolam und zusätzlich Clonazepam behandelt werden. Danach wird der Patient auf die Kinder-Intensivstation aufgenommen.

Neben der klinischen Untersuchung mit reduziertem Allgemeinzustand wird ein internistisch unauffälliger Befund beschrieben mit neurologisch regelrechten Pupillenreflexen und herabgesetzten Muskeleigenreflexen bei normalem Aufnahmelabor und unauffälligem CT des Kopfes.
Wegen erneuter generalisierter Krampfanfälle bekommt der Patient nochmals Clonazepam und Phenobarbital mit letztendlichem Sistieren der Krampfanfälle.

Am Folgetag wird nachmittags ein erneuter generalisierter Krampfanfall beobachtet. Das EEG zeigt links betonte SharpWaves im EEG. Ein zusätzlich angefertigtes MRT ergibt keinen richtungsweisenden Befund bezüglich der Ursache. Eine Liquoruntersuchung nach Lumbalpunktion unter Propofol-Narkose erbringt einen Normalbefund.

In der Nacht bekommt der Patient weitere epileptische Anfälle, weshalb bei relativer Therapieresistenz die Entscheidung zur kontinuierlichen Behandlung mit Thiopental fällt. Hierbei stellen sich akute allergische Reaktionen ein, die sich unter entsprechender intensiver Therapie bessern. Wegen der Therapie mit Hypnotika wird der Patient zudem intubiert und beatmet.
Danach wird bei weiterhin pathologischem EEG von Thiopental auf Propofol gewechselt, wobei zeitweise $10 \mathrm{mg} / \mathrm{kgKG} /$ $\mathrm{h}$ verabreicht werden.

Im weiteren Verlauf wird der Patient auf die Erwachsenen-Intensivstation verlegt, wo er mit Valproat in Kombination mit Levetiracetam und MethylprednisolonPulstherapie unter Verdacht auf eine autoimmunologisch vermittelte Enzephalopathie behandelt wird. Darüber hinaus erhält er Antibiotika und Norepinephrin bei Fieber und Kreislaufproblemen.

Vier Tage nach Klinikaufnahme entwickelt der Patient eine akute Nierenschädigung bei Rhabdomyolyse mit blutigem Urin und Anstieg der Muskelenzyme. Im Weiteren stellt sich dann der Verdacht auf ein Propofol-Infusionssyndrom mit zusätzlicher Critical-Illness-Polyneuropathie und Polymyopathie.

Nach insgesamt 7-wöchiger Intensivbehandlung kann der Patient in eine stationäre Rehabilitationsklinik verlegt werden.

\section{Beanstandung der ärztlichen Maßnahmen}

Die Verfahrensbevollmächtigte wünschte eine Begutachtung der Behandlung, ob diese den ärztlichen Standards entsprochen hätte, insbesondere im Hinblicke auf die Propofol-Unverträglichkeit und die daraus resultierenden Folgen wie „Muskelzerfall“, Narbe am Hals nach Tracheotomie und verzögerte Heilung mit Wiederholung eines Schuljahres.

\section{Stellungnahme des Klinikums}

Der Patient habe aufgrund seines andauernden und rezidivierenden Krampfgeschehens zunehmend mehr und auch kontinuierlich antiepileptische Medikamente erhalten. Schließlich sei aufgrund 
des relativ erfolglosen Einsatzes verschiedener Medikamente eine Thiopental-Narkose begonnen worden. Wegen allergischer Reaktionen habe der Patient daraufhin Propofol mit einer Maximaldosis von $9 \mathrm{mg} / \mathrm{kgKG} / \mathrm{h}$ erhalten. Die nachfolgende schwere Rhabdomyolyse und alle Folgeprobleme seien möglicherweise als Propofol-Infusionssyndrom zu werten. Das Propofol sei leitliniengerecht zur Behandlung des Status epilepticus eingesetzt worden; dafür habe man sich aufgrund der Unverträglichkeit von Thiopental entschieden.

\section{Gutachten}

\section{Pädiatrisches Gutachten}

Die Behandlung der erstmaligen Krampfanfälle mit statusartiger Häufung sei leitliniengerecht durchgeführt worden mit Midazolam, gefolgt von Clonazepam und Phenobarbital sowie Levetiracetam. Möglicherweise hätte man auch die ThiopentalNarkose vermeiden können bei entsprechender Höherdosierung von Levetiracetam. Die eingetretene allergische Reaktion sei leitlinienkonform behandelt worden.

Als Alternative sei dann Propofol gewählt worden, das zur Narkose und ab 17 Jahren auch zur Langzeitsedierung zugelassen sei. Es sei jedoch zu kritisieren, dass stattdessen nicht den Leitlinien entsprechend eine Kombination von Fentanyl- und Midazolam-Dauerinfusion begonnen worden sei mit Krampfdurchbrechung und EEG-Verbesserung durch Levetiracetam sowie Valproat, um die Langzeit-Propofol-Medikation zu vermeiden. Stattdessen sei eine immer höhere Propofol-Dosis gegeben worden mit ca. $10 \mathrm{mg} / \mathrm{kgKG} / \mathrm{h}$. Es sei bekannt, dass bei Langzeitanwendung von Propofol in Dosierungen von $>4 \mathrm{mg} /$ $\mathrm{kgKG} / \mathrm{h}$ das Risiko eines Propofol-Infusionssyndrom erheblich ansteige und diese Patienten wegen der dann auch eingetretenen Nebenwirkungen zu monitoren seien inkl. Leber-, Muskel- und Stoffwechselwerten. Eine Erhöhung der Laktatserumkonzentration sei irrtümlicherweise als sepsisassoziiert gewertet worden.

Trotz der beginnenden Rhabdomyolyse sei die Propofol-Medikation in einer deutlich über der für die Langzeitsedierung emp- fohlenen Dosis von $4 \mathrm{mg} / \mathrm{kgKG} / \mathrm{h}$ weitergeführt worden. Auch bei der Verlegung auf die Erwachsenen-Intensivstation sei nicht die Möglichkeit eines Propofol-Infusionsyndroms als Ursache der Verschlechterung des Allgemeinzustandes kommuniziert worden.

Bekannt sei auch, dass sich insbesondere in Kombination mit hochdosierten Steroiden wie Methylprednisolon und/oder Katecholaminen wie Norepinephrin das Risiko für ein Propofol-Infusionssyndrom erhöhe.

\section{Stellungnahmen zum Gutachten}

Zu dem Gutachten wurden ärztlicherseits folgende Einwendungen erhoben:

Dem Gutachten müsse widersprochen werden, insbesondere dass der Status epilepticus nicht leitliniengerecht therapiert worden sei. Die Leitlinien beider zuständiger ärztlichen Fachgesellschaften (Neuropädiatrie/Neurologie) würden eine Stufenbehandlung empfehlen, die sich an der Dauer des Status epilepticus orientiere: zunächst mit Benzodiazepinen, anschließend Levetiracetam bzw. Valproat (dieses Letztere jedoch fehlend in der pädiatrischen Leitlinie). In Stufe 3 fände sich neben Thiopental und Midazolam auch das Propofol in der Erwachsenenleitlinie.

Der Gutachter kritisiere die Nichtausschöpfung der Levetiracetam-Dosis. Ob dies wirklich zu einer Krampffreiheit geführt hätte, bleibe dahingestellt und werde bestritten.

Die Entscheidung für Propofol sei kein Verstoß gegen die Leitlinien gewesen. Der komplizierte Verlauf und die fehlende Effektivität der verschiedenen Antikonvulsiva hätten zur Entscheidung geführt, durch Narkoseinleitung die Krampfanfälle zu durchbrechen. So verbleibe Propofol in Stufe 3 als ein mögliches Antikonvulsivum.

Im vorliegenden Fall hätte eine Güterabwägung zwischen den Problemen eines Status epilepticus und dem möglichen Risiko eines Propofol-Infusionssyndroms bestanden. Aus der maßgeblichen Sicht ex ante sei ihres Erachtens nach das Vorgehen nicht zu beanstanden. Ob eine frühere Reduktion der Propofol-Dosis ein Pro-
pofol-Infusionssyndrom hätte verhindern können, sei ebenfalls fraglich.

Somit könne kein schuldhafter Behandlungsfehler festgestellt werden. Der Verlauf sei als „schicksalhaft“ zu bewerten.

\section{Anästhesiologisch-intensiv- medizinisches Gutachten}

Eine über Tage notwendige Intensivtherapie mit Analgosedierung und Beatmung wäre ohnehin notwendig gewesen aufgrund des Status epilepticus. Der Wechsel auf Propofol wird als standardgerecht bewertet einschließlich der höheren Dosierung. Levetiracetam und Phenobarbital hätten als Alternative zur Thiopental-Narkose höher dosiert werden können.

Der anästhesiologisch-intensivmedizinische Gutachter bewertet es als Behandlungsfehler, dass bei der für ein PropofolInfusionssyndrom typischen Symptomatik nicht rasch die Diagnose gestellt und die Infusion erst einen Tag später beendet wurde. Es sei schwer zu entscheiden, welche Komplikationen im Behandlungsverlauf auf den Fehler zurückzuführen seien. Wahrscheinlich wäre bei verkürzter Dauer der Propofol-Infusion das Multiorganversagen ebenfalls aufgetreten, jedoch milder verlaufen. Eine genaue Abgrenzung sei nicht möglich.

\section{Bewertung der Haftungsfrage}

Die Schlichtungsstelle schloss sich dem pädiatrischen Gutachten im Ergebnis an. Dem intensivmedizinisch-anästhesiologischen Gutachten konnte man sich im Hinblick auf die Fehlerfrage nicht anschließen, weil im Gutachten maßgebliche Literatur nicht beachtet wurde.

Die stufenweise eskalierende Vorgehensweise bei dieser komplexen Therapie geschah zunächst regelkonform, jedoch wurden die frühen Hinweise bei hoher Risikokonstellation für ein Propofol-Infusionssyndrom aus der Sicht ex ante nicht ausreichend berücksichtigt.

Propofol ist ein Hypnotikum mit guter Steuerbarkeit, schnellem Wirkungseintritt und kurzer Wirkdauer. Es gehört zu den 
am häufigsten eingesetzten Substanzen in der Anästhesie; außerdem wird es regelhaft bei Sedierungen für diagnostische Maßnahmen und auch zur Sedierung im intensivmedizinischen Bereich angewandt. Für das Medikament gelten spezielle Zulassungsbeschränkungen:

- Zur Anästhesie und Sedierung für diagnostische Prozeduren ist Propofol 1\% bei Kindern > 1 Monat und Propofol 2\% bei Kindern > 3 Jahre zugelassen.

- Zur Sedierung in der Intensivmedizin ist Propofol erst > 16 Jahren zugelassen, auch wenn es in der pädiatrischen Intensivmedizin weltweit entgegen den Vorgaben in der Fachinformation bis zu $24 \mathrm{~h}$ mit einer streng limitierten Dosierung ( $<4 \mathrm{mg} / \mathrm{kgKG} / \mathrm{h})$ angewandt wird.

- Zur intensivmedizinischen Sedierung von Erwachsenen ist Propofol bis zu 7 Tage und nur in einer Dosierung $<4 \mathrm{mg} / \mathrm{kgKG} / \mathrm{h}$ zugelassen und soll nur von anästhesiologisch oder intensivmedizinisch ausgebildeten Ärzten verabreicht werden.

Die Arzneimittelkommission der deutschen Ärzteschaft hat 2004 auf schwere Arzneimittelnebenwirkungen in Form des sog. Propofol-Infusionssyndroms hingewiesen. Begünstigende Risikofaktoren für das Entstehen eines Propofol-Infusionssyndroms sind schwere Erkrankungen wie z.B. Polytrauma, Schädel-Hirn-Trauma, Fieber, Katecholamin-Therapie, Mitochondriopathien in Verbindung mit langer und/oder hoher Propofol-Dosierung ( $>4 \mathrm{mg} / \mathrm{kgKG} / \mathrm{h}$ und $>48 \mathrm{~h}$ ). Die Letalität ist hoch und liegt nach seinerzeit publizierten Fallberichten (2006) bei $32 \%$.

Im vorliegenden Fall wurde fehlerhaft eine deutlich höhere Dosierung gewählt, die zeitweise im Bereich von $10 \mathrm{mg} / \mathrm{kgKG} / \mathrm{h}$ lag. Grundsätzlich soll nach $48 \mathrm{~h}$ Anwendung von Propofol die Indikation kritisch überprüft und eine Reduzierung der Dosierung in Erwägung gezogen werden. Auch dies ist bei dem Patienten fehlerhaft nicht erfolgt. Ebenfalls unterblieb eine differenzialdiagnostische Abklärung eines Propofol-Infusionssyndroms, obwohl ein schweres Krankheitsbild unter Einbeziehung des zentralen Nervensystems vorlag und über einen längeren Zeitraum Propofol in hoher Dosierung zugeführt wurde.
In der Zusammenschau der Fehler muss hier von einem schweren Behandlungsfehler ausgegangen werden, da durch die fehlerhaft zu hohe Dosierung des Propofol eine unnötig risikoreiche Situation geschaffen wurden, die dann wahrgenommen und auf die nicht zeitgerecht reagiert wurde.

Ein schwerer Behandlungsfehler setzt nicht nur einen eindeutigen Verstoß gegen bewährte ärztliche Behandlungsregeln oder gesicherte medizinische $\mathrm{Er}$ kenntnisse voraus, sondern erfordert auch die Feststellung, dass ein Fehler vorliegt, der aus objektiver Sicht nicht mehr verständlich erscheint, weil er einem Arzt schlechterdings nicht unterlaufen darf (BGH VersR 2001,115).

Unter Berücksichtigung der Umstände dieses Falles ist nach Einschätzung der Schlichtungsstelle von einem schweren Fehler auszugehen.

\section{Umkehr der Beweislast}

Damit stellt sich die Frage nach der Beweislastverteilung zwischen den Parteien:

Ein schwerer Behandlungsfehler, der generell geeignet ist, einen Schaden der tatsächlich eingetretenen Art herbeizuführen, führt grundsätzlich zu einer Umkehr der objektiven Beweislast für den ursächlichen Zusammenhang zwischen dem Behandlungsfehler und dem primären Gesundheitsschaden. Es ist nicht erforderlich, dass der grobe Behandlungsfehler die einzige Ursache für den Schaden ist. Es reicht aus, dass der grobe Behandlungsfehler generell geeignet ist, den eingetretenen primären Schaden zu verursachen; nahelegen oder wahrscheinlich braucht der Eintritt eines solchen Erfolges nicht zu sein (vgl. BGH VersR 2004, 909).

Ausgeschlossen ist eine Verlagerung der Beweislast auf die Behandlungsseite ausnahmsweise nur dann, wenn

- jeglicher haftungsbegründende Ursachenzusammenhang äußerst unwahrscheinlich ist oder

- sich nicht das Risiko verwirklicht hat, dessen Nichtbeachtung den Fehler als grob erscheinen lässt (vgl. BGH VersR 2004, 909) oder
- der Patient durch sein Verhalten eine selbstständige Komponente für den Heilungserfolg vereitelt hat und dadurch in gleicher Weise wie der grobe Behandlungsfehler des Arztes dazu beigetragen hat, dass der Verlauf des Behandlungsgeschehens nicht mehr aufgeklärt werden kann (vgl. OLG Braunschweig VersR 1998,459).

Eine solche Ausnahmesituation lag hier nicht vor.

Die Beweislastumkehr bezieht sich auf folgende Primär- und typischerweise damit verbundene sekundäre Gesundheitsschäden: Propofol-Infusionssyndrom mit Rhabdomyolyse, Multiorganversagen, CriticalIllness-Neuropathie und -Myopathie sowie die damit verbundenen Beeinträchtigungen.

\section{Autorinnen/Autoren}

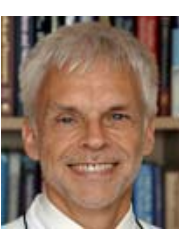

Thomas Hachenberg

Prof. Dr. Dr. med. Facharzt für Anästhesiologie und Intensivmedizin. Ärztliches Mitglied der Schlichtungsstelle für Arzthaftpflichtfragen der norddeutschen Ärztekammern. Direktor der Klinik für Anästhesiologie und Intensivtherapie am Universitätsklinikums Magdeburg A.ö.R.

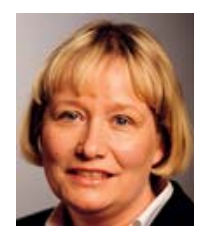

\section{Christine Wohlers}

Rechtsanwältin der Schlichtungsstelle für Arzthaftpflichtfragen der norddeutschen Ärztekammern

\section{Korrespondenzadresse}

Prof. Dr. Dr. med. Thomas Hachenberg

Klinik für Anästhesiologie und

Intensivtherapie

Universitätsklinikum Magdeburg A. ö. R.

Leipziger Straße 44

39120 Magdeburg

thomas.hachenberg@med.ovgu.de

\section{Bibliografie}

DOI https://doi.org/10.1055/a-0978-7685 Anästhesiol Intensivmed Notfallmed Schmerzther 2019; 54: 572-574 @ Georg Thieme Verlag KG Stuttgart · New York | ISSN 0939-2661 